\title{
USO POTENCIAL DE LA MANZANILLA MATRICARIA CHAMOMILLA L. Y EXPERIENCIAS EN NICARAGUA
}

\section{POTENTIAL USE OF CHAMOMILLA L. MATRICARIA MANZANILLA AND EXPERIENCES IN NICARAGUA}

\author{
Liliana Elisabet Meza Peter ${ }^{1}$ \\ Luis María Dicovskiy Riobóo ${ }^{2}$
}

(Recibido/received: 9-marzo-2020; aceptado/accepted: 20-Mayo-2020)

\begin{abstract}
RESUMEN: Con esta revisión bibliográfica se quiere aportar al uso y diversificación de la manzanilla, Matricaria chamomilla L., la cual es una hierba aromática con múltiples usos y muy difundida. Esta planta no es exigente con respecto a la calidad de los suelos, y es una especie plástica que se adapta a diversos climas. En Nicaragua es parte de la etnobotánica tradicional del país. Entre los usos potenciales de la especie están su uso medicinal por sus propiedades: antiinflamatoria, espasmolítica, antiulcerosa, carminativa, digestiva, bactericida, fungicida y sedante suave. Para cosméticos como champú o enjuague capilar o en cremas para suavizar y regenerar la piel. Y también como insecticida ecológico.
\end{abstract}

PALABRAS CLAVE: Matricaria chamomilla, hierba aromática, etnobotánica

ABSTRACT: With this literature review we want to contribute to the use and diversification of chamomile, Matricaria chamomilla L., which is an aromatic herb with multiple uses and widespread. This plant is not demanding with respect to the quality of the soils, and is a plastic species that adapts to various climates. In Nicaragua it is part of the traditional ethnobotany of the country. Among the potential uses of the species are its medicinal use for its properties: antiinflammatory, spasmolytic, antiulcer, carminative, digestive, bactericidal, fungicidal and mild sedative. For cosmetics such as shampoo or hair rinse or in creams to soften and regenerate the skin. And also as an ecological insecticide.

KEYWORDS: Matricaria chamomilla, aromatic herb, ethnobotany

\footnotetext{
${ }^{1}$ Facultad de Veterinaria de Zaragoza, España.Imeza@unizar.es

${ }^{2}$ Sede Regional UNI-Norte, Nicaragua. luis.dicovskiy@norte.uni.edu.ni
} 


\section{Liliana E. Meza P.; Luis M. Dicovskiy R.}

\section{INTRODUCCIÓN}

En el mundo el mercado de exportaciones de especies aromáticas es mayor a 770 millones de dólares (León León \& Olaya Prado, 2015). La manzanilla, Matricaria chamomilla L., sinónimo Matricaria recutita, es una hierba aromática con múltiples usos y muy difundida en América y Europa. En Nicaragua la manzanilla es parte de la etnobotánica tradicional del país, aunque su cultivo se hace en pequeña escala. Las zonas de mayor producción, tradicionalmente son: El Valle de la Laguna, Estelí y Wiwilí, Jinotega (IICA - CEDEMETRA, 2005)

La manzanilla ha sido consumida tradicionalmente en Nicaragua en forma de infusión de sus flores y planta. Se ha utilizado tradicionalmente en casos de dolor de estómago, cólicos, gases intestinales, sarpullido, sarampión, alergia, retrasos menstruales, alteraciones nerviosas y mal de ojo (Mancheno, 1994). También se ha reportado su uso en el tratamiento de posologías veterinarias en el Norte de Nicaragua (González-Castillo, y otros, 2019).

Con esta revisión bibliográfica sobre el uso de la manzanilla, se quiere aportar elementos a la diversificación y procesamiento de cultivos locales, aportando referencias de una especie ampliamente aceptada por la etnobotánica tradicional en Nicaragua.

\section{DESARROLLO}

Nombre común Manzanilla , Especie: Matricaria chamomilla L. sinónimo Matricaria recutita, Familia: Asteraceae / Compositae.

Botánica, Ecología. Matricaria chamomilla es Nativa de Eurasia. Es una hierba anual, de unos $60 \mathrm{~cm}$ de altura. Su Inflorescencia está compuesta de cabezuelas solitarias a agrupadas por varias en el extremo de las ramas (Vibrans, Hanan Alipi, \& Mondragón Pichardo, 2009). Originaria de Europa, difundida en forma natural o por cultivo en Europa: España, Yugoslavia y Hungría, cuenca del Danubio; el norte de África: Egipto, oeste de Asia: Turquía y América: Argentina, U.S.A. y Venezuela (Camaroni, 2010). M. chamomilla es común como especie arvense en los cultivos de cereales en la Comunidad de Aragón en España (Cirujeda, Zaragoza, \& Aibar, 2008).

La manzanilla no es exigente con respecto a la calidad de los suelos, y es una especie plástica que se adapta a diversos climas, aunque mayor rendimiento y mejor calidad se obtienen en clima templado a templado-cálido, temperatura media anual entre 15 y $23^{\circ} \mathrm{C}$ y condición de subhúmedo (Rubio, 2004).

Cultivo: Como la semilla es muy pequeña se requiere una preparación esmerada del suelo, para la siembra, lo común es usar 8 a $10 \mathrm{~kg} / \mathrm{ha}$ de semilla, y se puede usar sembradoras de semillas pequeñas de pasturas, con surcos a $30 \mathrm{~cm}$. El momento en que se debe entrar a cosechar es cuando la floración se manifiesta plena, con la mayoría de los capítulos abiertos; el lote se presenta como un manto blanco. Lo común es que un cultivo se cosecha dos veces. En

El Higo Revista Científica / Volumen 10. No. 01, pp. 1-8 / Junio 2020 
campo de mediana fertilidad, bien trabajados, se puede esperar un rendimiento de $1.700 \mathrm{~kg} / \mathrm{ha}$ de material fresco (flores), en el primer corte y $800 \mathrm{~kg}$ en el segundo (Rubio, 2004).

Se suele cosechar las flores a mano en las horas centrales del día, también se puede cosechar con máquina. En el caso de la flor seca, el secado se hace a la sombra (Del Monte Maíz, Rico Jiménez, Hernández Rodríguez, \& Navazo Bartolomé, 2014).

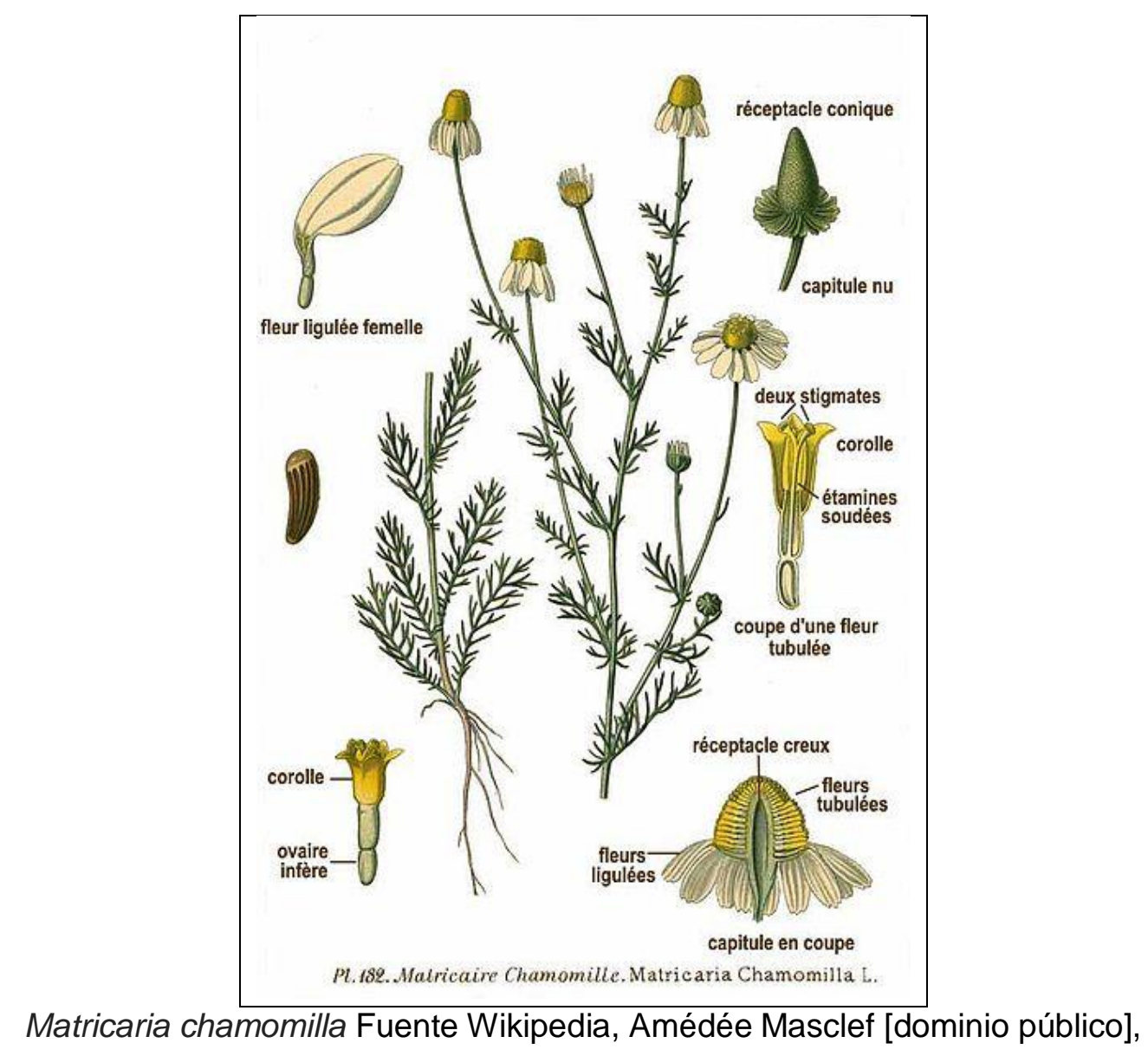

Usos: En Nicaragua Salinas y Grijalva (1994) reportan su uso para diarreas, baños y problemas digestivos. En el libro de planta medicinales editado por el ICCA - CEDEMTRA, se expresa que la manzanilla posee "acción antiinflamatoria, espasmolítica, antiulcerosa, carminativa, digestiva, bactericida, fungicida y sedante suave, debido a los diferentes principios activos de la droga. El(-)-alfa-bisabolol, camazuleno,matricina, espiroéteres y flavonoides son los principales responsables de la acción antiinflamatoria" (IICA - CEDEMETRA, 2005, pág. 29).

La manzanilla tiene varios usos: medicinales, alimenticios y cosméticos. El extracto hidroalcohólico de la flor de manzanilla se usa como antiinflamatorio y antipirético, musculotrópico espasmolítico, ansiolítico, vulnerario, desodorante, antibacteriano y estimulante del metabolismo de la piel (Meneses-Reyes, Soto-Hernández, Espinosa-Solares, \& Ramírez-Guzmán, 2008). 


\section{Liliana E. Meza P.; Luis M. Dicovskiy R.}

La Manzanilla se ha usado en productos cosméticos por ejemplo su aceite, el extracto de flor/ hoja; el agua de flor para hacer: vapores faciales, cuidado de la piel y el cabello; champú y enjuague capilar para cabellos claros (Shital, Aishwarya, Amar, \& Ravindra, 2013). En la cosmética se ha utilizado tradicionalmente en el tratamiento de las pieles sensibles y secas ya que ayuda a suavizar la piel y promueve la regeneración celular (Devia Pineda, 2007).

En las flores de planta de manzanilla es donde se encuentran la mayor concentración de sus componentes activos, los cuales entre otros son aceites esenciales como el bisabolol y la apigenina; los cuales tienen actividad espasmolítica. Se comprobó de forma experimental que el extracto fluido al $30 \%$ de $M$. recutita tiene acción espasmolítica, en el músculo liso intestinal y en general lo hace de manera similar a la papaverina, droga de referencia (Morón Rodríguez, Furones Mourelle, \& Pineda Gutiérrez, 96).

La leishmaniasis cutánea, es endémica en Nicaragua, y está asociada a hábitats silvestres (Darce, y otros, 1991). En una investigación in vitro donde se evaluó aceites esenciales de Matricaria chamomilla en el control de Leishmania. Los resultados aportaron bases para sugerir que el aceite esencial de manzanilla tiene potencial para el desarrollo de medicamentos contra Leishmania (Ríos R., y otros, 2008).

Se investigó el efecto de $M$. chamomilla sobre el desarrollo de la dependencia de morfina y la expresión de la abstinencia en ratas mediante la administración conjunta crónica de extracto de manzanilla con morfina. Los resultados sugieren que el extracto de $M$. chamomilla inhibe el desarrollo de la dependencia de morfina y la expresión del síndrome de abstinencia (Gomaa, Hashem, Mohamed, \& Ashry, 2003).

M. chamomilla es un antioxidante natural con propiedades de hepatoprotector. Este efecto fue evaluado en ratas y se observó efectos beneficiosos de sus extractos acuosos, en la protección del daño hepático oxidativo producido por intoxicación del herbicida Paraquat (Tavakol, y otros, 2015).

Los extractos de $M$. chamomilla también tienen propiedades insecticidas, estos se han usado para controlar la chinche de los eucaliptus, Thaumastocoris peregrinus (Hemipetra: Thaumastocoridae), pero además se comprobó que su aplicación no afecta a su principal parasitoide. Cleruchoides noackae (Hymenoptera: Mymaridae), lo que indica que puede ser una opción ecológica para controlar esta chinche (Haas, y otros, 2018). Daza y Florez (2006) sugieren el uso de sus aceites esenciales en la piel, como repelente de mosquitos.

Entre sus aplicaciones veterinarias se destacan sus propiedades en la desinflamación y cicatrización de heridas, Castro Torres (2015), demostró que la infusión de manzanilla al 4\% es una alternativa de fácil acceso para la desinflamación y cicatrización de heridas ocasionadas por orquiectomía y castrado, de lechones. 
Otro potencial usa de los extractos de M. chamomilla es como conservantes de cosméticos. Esto es debido a sus propiedades bactericidas (Herman, Herman, Domagalska, \& Młynarczyk, 2013).

Industrialización y comercialización: El cultivo de manzanilla se incluye en las denominadas genéricamente como Plantas Aromáticas y Medicinales (PAM). En Nicaragua, no existen datos estadísticos sobre la superficie cultivada de manzanilla, sin embargo está problemática es común en otros países debido a la dispersión y a la gran diversidad de especies botánicas y modelos productivos que dificultan la recopilación de datos de éste "micro sector" (Pascual, 2018).

El mercado de la manzanilla tiene varias salidas, en función del producto final: Planta viva y conservada (liofilizada), como plantas ornamentales; flor fresca, seca o congelada para fitomedicamentos (humano 0 animal); aceites esenciales (perfumería, cosmética, ambientadores); extractos (farmacéutico-medicinal y aditivos para la industria alimentaria) El gráfico siguiente describe el flujo de transformación general de las materias primas a base de plantas aromáticas y medicinales (Moré, 2009):

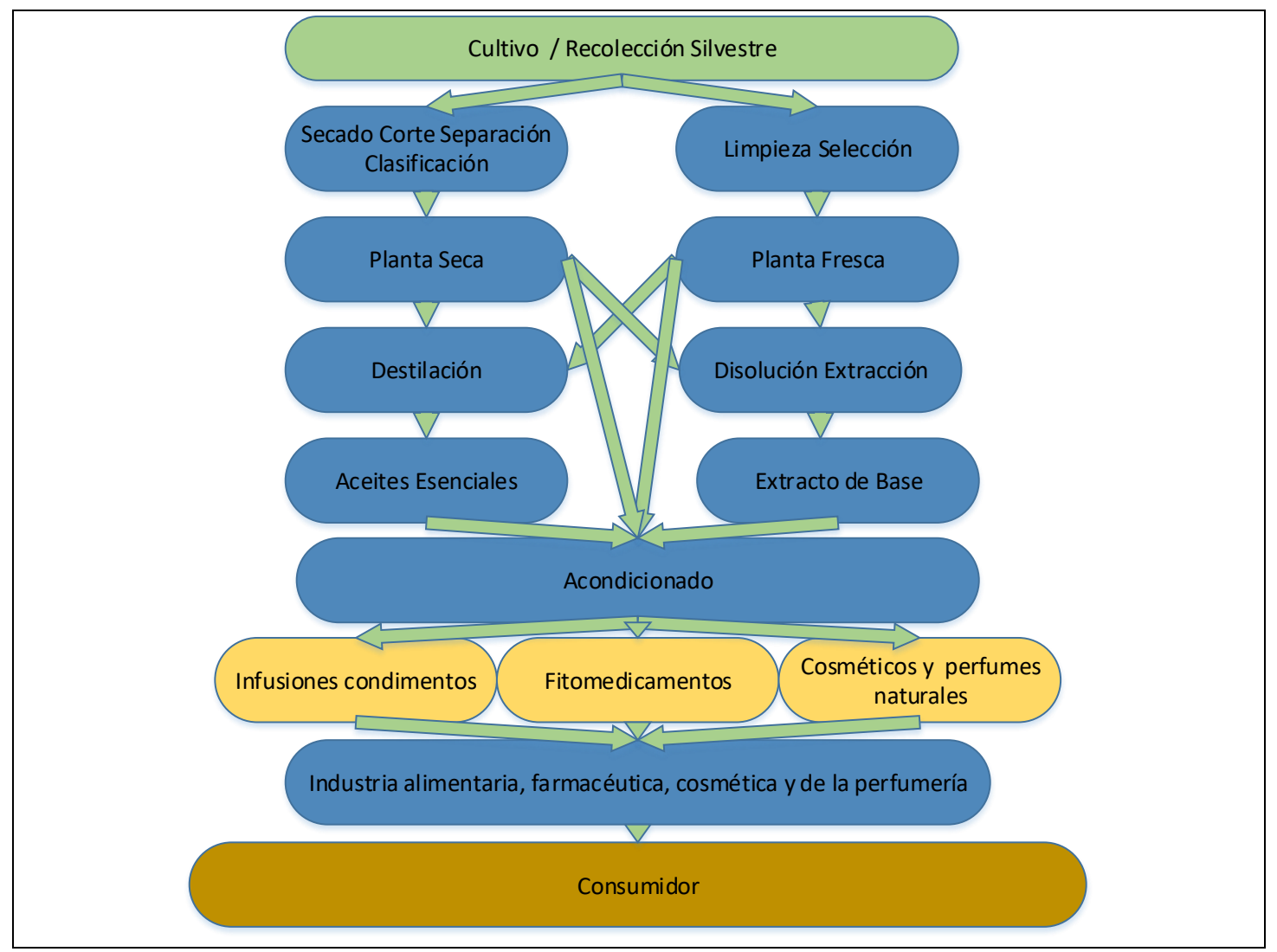

Flujo de transformación general de las materias primas a base de PAM, Adaptado de Moré, E. (2009) 


\section{Liliana E. Meza P.; Luis M. Dicovskiy R.}

En Nicaragua, en la actualidad la comercialización de la manzanilla de producción nacional se realiza fundamentalmente como planta o flor seca en mercados, y en menor volumen procesada en bolsitas filtrantes para infusiones. Mientras que los productos con mayor transformación derivados de la manzanilla provienen del mercado externo.

\section{CONCLUSIONES}

La manzanilla tiene múltiples usos, como medicinales, alimenticios y cosméticos El cultivo de manzanilla es relativamente sencillo y tiene un gran potencial como materia prima para la industria local o para la exportación de sus principios activos. Actualmente es un cultivo poco extendido en Nicaragua, considerando que existen condiciones para su cultivo resulta una alternativa productiva interesante. Sin embargo, la necesidad de estructura para transformar y comercializar el producto debe ser considerado como una necesidad en el caso de fomentar este cultivo.

\section{REFERENCIAS}

Camaroni, M. G. (2010). Ficha técnica manzanilla. (Matricaria recutita) cadena hierbas aromáticas y especies. Buenos Aires: Ministerio de Agricultura Ganadería y Pesca, Gobierno de la Nación.

Castro Torres, S. (2015). Evaluación del efecto desinflamatorio y cicatrizante de 3 diferentes concentraciones de una infusión de manzanilla (Matricaria chamomilla l.) vía tópica, en orquiectomía de lechones. Thesis, Universidad de San Carlos, Guatemala.

Cirujeda, A., Zaragoza, C., \& Aibar, J. (2008). Factores que influyen en la biodiversidad de la flora arvense de los cereales. VIII Congreso SEAE Bullas, (pág. 8). Murcia. Obtenido de https://citarea.cita-aragon.es/citarea/bitstream/10532/3132/1/czl365.pdf

Darce, M. M., Palacios, X., Belli, A., Gomez-Urcuyo, F., Zamora, D., \& Grimaldi Jr, G. (1991). Etiology of human cutaneous leishmaniasis in Nicaragua. Transactions of the Royal Society of Tropical Medicine and Hygiene, 85(1), 58-59.

Daza, L., \& Florez, N. (2006). Diseño de un repelente para insectos voladores con base en productos naturales. Tesis de grado, Universidad EAFIT, Escuela de Ingeniería. Obtenido de https://repository.eafit.edu.co/bitstream/handle/10784/354/LeticiaPaulina_DazaM_2006.p $\mathrm{df}$ ? sequence $=1$ \&isAllowed $=\mathrm{y}$

Del Monte Maíz, M., Rico Jiménez, J. C., Hernández Rodríguez, O., \& Navazo Bartolomé, A. R. (2014). Estudio etnoecológico de la manzanilla( Matricaria recutita l.) en el Oso, Ávila. Obtenido de http://www.conama.org/conama/download/files/conama2014/CT\%202014/Paneles/1896 711857_panel.pdf

del Rosal Rabesa, T., Baquero-Artigaob, F., \& García Miguelb, M. (2010). Leishmaniasis cutánea. Rev Pediatr Aten Primaria, 12(46), 263-271. Obtenido de http://scielo.isciii.es/pdf/pap/v12n46/revision1.pdf

Devia Pineda, J. (2007). Desarrollo de nuevos productos. Cuadernos de Investigación(59), 97. 
Gomaa, A., Hashem, T., Mohamed, M., \& Ashry, E. (2003). Matricaria chamomilla extract inhibits both development of morphine dependence and expression of abstinence syndrome in rats. Journal Pharmacological Sciences, 92(1), 50-55. Obtenido de https://search.proquest.com/docview/925340647?accountid=168360

González-Castillo, K. A., Chavarría-Rodríguez, R. J., Iglesias-Olivas, M. I., Rodríguez-Flores, O. R., Arcocha-Gómez, E., \& González-Valdivia, N. (2019). Plantas Utilizadas en Terapia Veterinaria en San Rafael del Norte, Jinotega, Nicaragua. En W. Cetzal-Ix, F. CasanovaLugo, A. J. Chay-Canul, \& J. F. Martínez-Puc, Agroecosistemas Tropicales (pág. 533). Campeche, México: Tecnológico Nacional de México.

Haas, J., Barbosa, L. R., Potrich, M., Lozano, E. R., Vismara, E. S., Baungratz, A. R., \& Mazaro, S. M. (2018). Toxicity assessment of plant extracts to cleruchoides noackae lin and huber (hymenoptera: Mymaridae). Agroforestry Systems, 1-9. doi:10.1007/s10457-0180242-6

Herman, A., Herman, A. P., Domagalska, B. W., \& Młynarczyk, A. (2013). Essential oils and herbal extracts as antimicrobial agents in cosmetic emulsion. Indian Journal of Microbiology, 53(2), 232-237. doi:10.1007/s12088-012-0329-0

IICA - CEDEMETRA. (2005). Plantas medicinales y otra especies útiles. Managua: IICA. Obtenido de http://www.renida.net.ni/renida/iica/f60-c389.pdf

León León, C. G., \& Olaya Prado, V. M. (2015). Implementación de un programa de capacitación sobre el mercado de manzanilla y su impacto en la producción y el bienestar de los agricultores del distrito de OTUZCO - 2015. Tesis de grado, Universidad Privada Antenor Orrego, Trujillo. Obtenido de http://repositorio.upao.edu.pe/bitstream/upaorep/1499/1/Leon_Leon_Programa_Capacita cion_Produccion.pdf

Mancheno, M. (1994). La "manzanilla " (Matricaria recutüa, Asteraceae) dentro del plan terapeútico del tratamiento de la enfermedad diarreica. En P. S. económica, Las plantas y el hombre (pág. 436). Quito: ABYA-YALA.

Meneses-Reyes, J. C., Soto-Hernández, R., Espinosa-Solares, T., \& Ramírez-Guzmán, M. (2008). Optimización del proceso de extracción de flavonoides de flor de manzanilla (Matricaria recutita L.). Agrociencia, 42(4), 425-433. Obtenido de http://www.scielo.org.mx/pdf/agro/v42n4/v42n4a5.pdf

Moré, E. (2009). Mercado y Comercialización de plantas aromáticas y medicinales. Solsona, Cataluña: INTRADER. Obtenido de http://pam.ctfc.es/docs/ficha\%20COMERCIALIZACION.pdf

Morón Rodríguez, F., Furones Mourelle, J., \& Pineda Gutiérrez, Z. (96). Actividad espasmolitica del extracto fluido de Matricaria recutita (manzanilla) en órganos aislado. Rev Cubana Plan Med, 1(1), 19-24. Obtenido de http://scielo.sld.cu/pdf/pla/v1n1/pla06196.pdf

Pascual, A. (29 de Agosto de 2018). Cultivo de aromáticas, buenas perspectivas ante el aumento de la demanda mundial. Obtenido de EFE Agro: https://www.efeagro.com/noticia/aromaticas/

Ríos R., Y. K., Otero J., A. C., Muñoz H., D. L., Echeverry R., M., Robledo R., S. M., \& Yepes C., M. A. (2008). Actividad citotóxica y leishmanicida in vitro del aceite esencial de manzanilla (Matricaria chamomilla). Rev. Colomb. Cienc. Quím. Farm., 37(2), 200-211. 
Rubio, M. (2004). Cultivo, industrialización y comercialización de la manzanilla (Matricaria recutita l.). Obtenido de Instituto Nacional de Tecnología Agropecuaria- INTA - Castelar: http://www.herbotecnia.com.ar/c-biblio010-21.html

Salinas, B. E., \& Grijalva, A. (1994). Diagnóstico de Nicaragua. En R. Ocampo, Domesticación de plantas medicinales en Centroamérica (pág. 132). Turrialba: CATIE.

Shital, G. S., Aishwarya, J. J., Amar, Z. G., \& Ravindra, S. B. (2013). A Review on Oils used in Herbal Cosmetics. Research Journal of Topical and Cosmetic Sciences, 4(2), 61-64. Obtenido de https://search.proquest.com/docview/1541981429?accountid=168360

Tavakol, H. S., Fariba, M., Abdolkarim, C., Hassan, G., Seyed-Mostafa, H., \& Akram, R. (2015). Hepatoprotective effect of matricaria chamomilla. $L$ in paraquat induced rat liver injury. Drug Research, 65(2), 61-64. doi:10.1055/s-0033-1363999

Vibrans, H., Hanan Alipi, A. M., \& Mondragón Pichardo, J. (14 de Julio de 2009). Asteraceae = Compositae, Matricaria recutita L. Obtenido de Malezas de Mexico: http://www.conabio.gob.mx/malezasdemexico/asteraceae/matricariarecutita/fichas/ficha.htm

\section{SEMBLANZA DE LOS AUTORES}
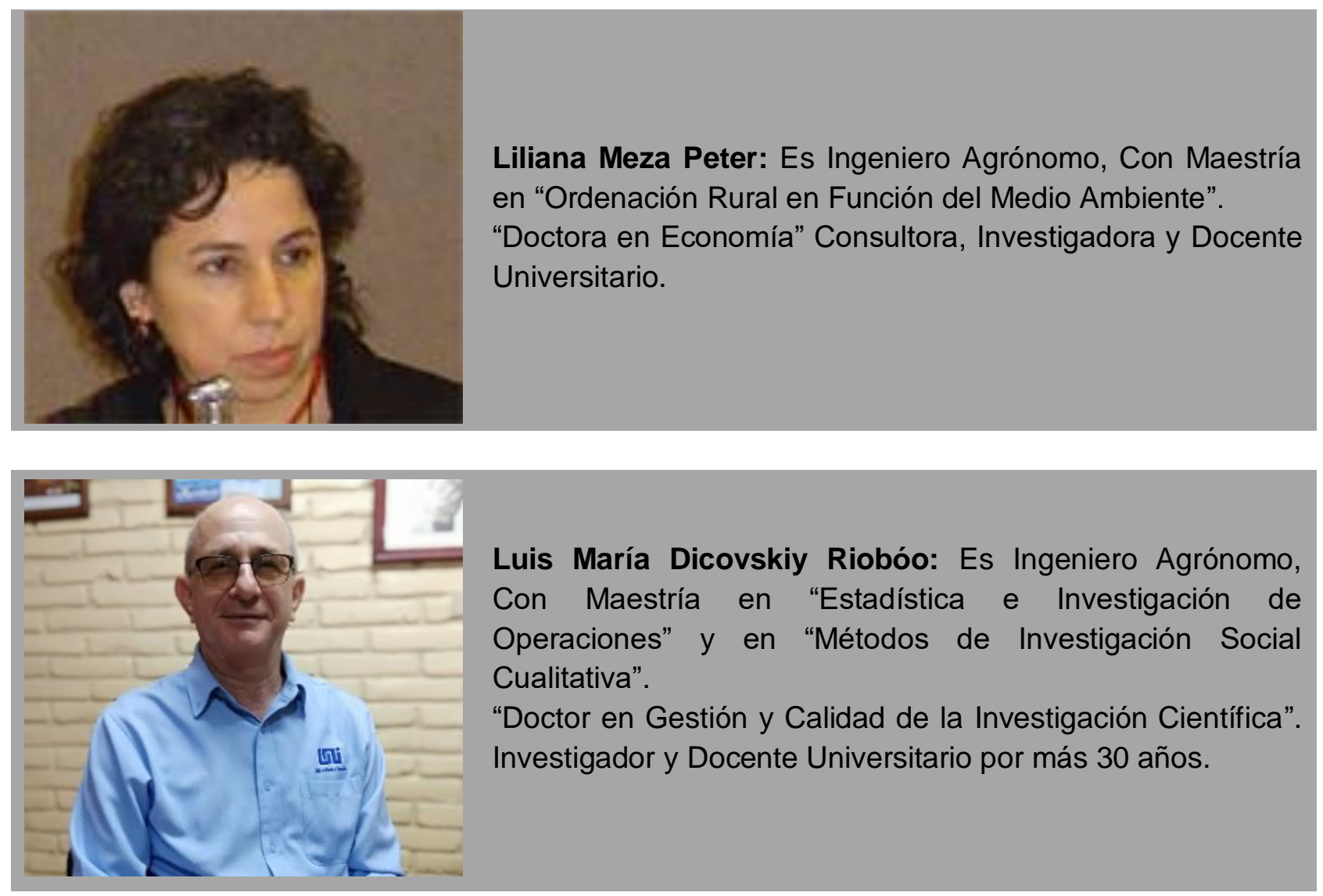